\title{
Hawking Temperature and String Scattering off the $2+1$ Black Hole
}

\author{
by \\ Kazuo Ghoroku ${ }^{1}$ \\ The Niels Bohr Institute, Blegdamsvej 17, Dk-2100 Copenhagen $\varnothing^{2}$ \\ and \\ Arne L. Larsen ${ }^{3}$ \\ Nordita, Blegdamsvej 17, Dk-2100 Copenhagen $\varnothing$ \\ May 7, 2019 \\ ${ }^{1}$ e-mail: ghoroku@nbivax.nbi.dk \\ ${ }^{2}$ Permanent address: Department of Physics, Fukuoka Institute of Technology, \\ Wajiro, Higashiku, Fukuoka 811-02, Japan. e-mail: gouroku@dontaku.fit.ac.jp \\ 3 e-mail: allarsen@nbivax.nbi.dk
}




\begin{abstract}
The $2+1$ black hole anti de Sitter solution, obtained as a special limit of the conformally exact $S L(2, R) \otimes S O(1,1) / S O(1,1)$ coset WZW model to all orders in $1 / k$, is investigated with respect to tachyon scattering. We calculate the off-shell reflection and transmission coefficients and we find an expression for the Hawking temperature, which in a certain limit reduces to the statistical result.
\end{abstract}




\section{Introduction}

A new way to obtain singular background configurations as the solutions of string equations has been developed by using gauged WZW models [1], especially in low space-time dimensions $(D<4)$. Although they are toy models of the realistic four dimensional theory, it is possible to study the scattering of string fields off these non-trivial black "species"; black holes, black branes e.t.c. Among those configurations, $2+1$ dimensional black hole anti de Sitter spacetime [2] has been examined according to the method developed in reference [1], and also by the duality transformation of a $2+1$ black string [3, 4]. But both procedures preserve the conformal invariance only at the leading order in the $\alpha^{\prime}$ expansion. A possible way to extract the conformally exact background configurations for metric and dilaton is, however, known [5].

The purpose of this letter is to study the $2+1$ dimensional black hole solution according to the procedure of [5] with the limiting process of the gauging parameters [6]. We first review the construction of the $2+1$ dimensional black string and discuss the limiting process by which it is transformed into the $2+1$ dimensional black hole anti de Sitter spacetime. We then solve the off-shell tachyon field equation in this background, and we obtain the reflection and transmission coefficients of the scattering of the tachyon off the black hole. We also obtain an expression for the Hawking temperature, which in a certain limit reduces to the statistical (thermal) value. The thermodynamical properties of the black hole are thus clarified.

\section{$2 \quad 2+1$ Black Hole Solution}

The black string solution was first obtained by Horne and Horowitz [3], using the method originally developed by Witten [1]. The idea is as follows: the startingpoint is the WZW model of a group $G$,

$$
S(g)=\frac{k}{4 \pi} \int_{\Sigma} \operatorname{Tr}\left(g^{-1} \partial_{+} g g^{-1} \partial_{-} g\right)-\frac{k}{12 \pi} \int_{B} \operatorname{Tr}\left(g^{-1} d g \wedge g^{-1} d g \wedge g^{-1} d g\right),
$$


where $g \in G$ and $\partial B=\Sigma$. After gauging an appropriate subgroup $H$ of $G$ by introducing the corresponding gauge fields, the gauge fields are integrated out and the target space metric and antisymmetric tensor can be read off by rewriting the action to the corresponding $2 \mathrm{D} \sigma$-model on the world sheet. Finally a dilaton field has to be included to ensure the vanishing of the $\beta$ function. This procedure guarantees the correct target space metric only at the semi-classical level, which here corresponds to the limit $k \rightarrow \infty$ (first order in $1 / k)$, so quantum corrections are generally expected to arise.

In the exact treatment of the theory, valid to all orders in $1 / k$, it is convenient to proceed in a somewhat different way [5]. Consider the effective action in target space:

$$
S(T)=\int d^{n} X \sqrt{-G} e^{\Phi}\left(G^{\mu \nu} \partial_{\mu} T \partial_{\nu} T-2 T^{2}+\ldots\right),
$$

where $\Phi$ and $G_{\mu \nu}$ are the dilaton and target space metric for the coset model under consideration. The dots denote the possible higher order terms in $T(X)$ as well as terms representing other string fields. The next step is to identify the linearised equation of motion of $T(X)$, which can be obtained from eq. (2), and the Virasoro constraint which determines the conformal weight of $T(X)$. Then we obtain:

$$
\left(L_{0}+\bar{L}_{0}\right) T=-\frac{1}{e^{\Phi} \sqrt{-G}} \partial_{\mu} G^{\mu \nu} e^{\Phi} \sqrt{-G} \partial_{\nu} T,
$$

where $L_{0}, \bar{L}_{0}$ are the Virasoro operators of $G / H$. The Virasoro operators, in turn, can be written in terms of the Casimir operators for the group $G$ and subgroup $H$, and they are expressed by the differential operators on the group parameter space. Since the Casimir operators contain single and double derivatives, one can read off the exact metric and dilaton by identifying the single and double derivatives on the two sides of eq. (3). But we can not obtain the exact form of the anti-symmetric tensor in this procedure. However, we do not need it in the analysis hereafter.

Taking $G=S L(2, R) \otimes S O(1,1)$ and $H=S O(1,1)$, the axial gauging leads to the following results:

$$
\begin{aligned}
d s^{2} & =\frac{2(k-2)}{(1-2 / k)(u v-1)-\rho^{2}-2 / k}\left[-\frac{1}{4} \frac{\rho^{2}+2 / k}{u v-1}(v d u+u d v)^{2}+\left(1+\rho^{2}\right) d u d v\right] \\
& +2 k_{1}\left[1+\frac{\rho^{2}}{(1-2 / k)(u v-1)-\rho^{2}}\right] d r^{2}
\end{aligned}
$$




$$
e^{\Phi}=(1-u v) \sqrt{\left[1+\rho^{2}+\left(\rho^{2}+2 / k\right) \frac{u v}{1-u v}\right]\left[1+\rho^{2}-2 / k+\rho^{2} \frac{u v}{1-u v}\right]} .
$$

Here the notations of the group variables, $u, v \in S L(2, R)$ and $r \in S O(1,1)$, are according to reference [7]. The parameter $\rho$ is defined as, $\rho^{2}=\eta^{2} k_{1} / k$, where $k$ and $k_{1}$ are the levels of $S L(2, R)$ and $S O(1,1)$, respectively, while $\eta$ is an "external" parameter which controls the ratio of the embedding of the $S O(1,1)$ subgroup to $S L(2, R)$ and $S O(1,1)$. In the axial gauge, the coordinate $r$ is related to one of the other (except $u, v$ ) parameters of $S L(2, R)$. In the limit of $\rho^{2}=\infty$, the gauging of $S L(2, R)$ is turned off and the coordinates are written by using the three parameters of $S L(2, R)$ only. The central charge is:

$$
c\left(\frac{S L(2, R) \otimes S O(1,1)}{S O(1,1)}\right)=\frac{3 k}{k-2},
$$

so that $c=26$ is obtained for $k=52 / 23$. Eqs.(4) and (5) are the exact form of the black string solution.

In order to obtain the black hole solution, we take the parametrization of the coordinates in the region $u v<0$ :

$$
u=\sinh R e^{t}, \quad v=-\sinh R e^{-t}, \quad r=\eta x .
$$

Then eqs. (4)-(5) become:

$d s^{2}=2(k-2)\left[d R^{2}-\frac{\left(1+\rho^{2}\right) d t^{2}}{\left(1+\rho^{2}\right) \operatorname{coth}^{2} R-\rho^{2}-2 / k}+\frac{\rho^{2} \operatorname{coth}^{2} R d x^{2}}{\left(\rho^{2}+1-2 / k\right) \operatorname{coth}^{2} R-\rho^{2}}\right]$,

and:

$$
e^{\Phi}=\cosh ^{2} R \sqrt{\left[1+\rho^{2}-\left(\rho^{2}+2 / k\right) \tanh ^{2} R\right]\left[1+\rho^{2}-2 / k-\rho^{2} \tanh ^{2} R\right]} .
$$

Before coming to the black hole we notice that the usual black string form is obtained by the further change of coordinates:

$$
t=\sqrt{\frac{2}{k\left(1+\rho^{2}\right)}} t^{\prime}, \quad x=\frac{\rho}{k-2} \sqrt{2 k} x^{\prime}, \quad \sinh ^{2} R=\frac{k r^{\prime}-k \sqrt{\frac{2}{k-2}}\left(\rho^{2}+1\right)}{\sqrt{2(k-2)}} .
$$


Next we consider the limit of $\rho^{2}=\infty$, in order to obtain the black hole solution. In this limit the transformations (10) are not well-defined, so we take the change of variable, $\hat{r}=\cosh R$, only. Then we get:

$$
d s^{2}=2(k-2)\left[-\left(\hat{r}^{2}-1\right) d t^{2}+\frac{d \hat{r}^{2}}{\hat{r}^{2}-1}+\hat{r}^{2} d x^{2}\right], \quad \Phi=\text { const } .
$$

This limiting process effectively decouples $S L(2, R)$ in the gauging [3, 6, 7]. Therefore, in this limit the three group variables of $S L(2, R)$ are kept to represent the space-time, while the parameter of $S O(1,1)$ has been dropped. In the opposite limit $\rho^{2}=0$, the gauging is done for $S L(2, R)$ only. Then we obtain the solution of the 2D black hole, which is obtained in [1], and an independent one-dimensional space $R$. We can see further that the limiting processes produce no new solutions in the case of the vector gauging. This is because the parameters of $S L(2, R)$ and $S O(1,1)$ are not combined in the vector gauging.

Solution (11) is exactly the $(M=1, J=0)$ version of the $2+1$ dimensional black hole anti de Sitter spacetime, found by Bañados et. al. [2], if we periodically identify $x=x+2 \pi$. The full two-parameter family of $(M, J)$ black holes is obtained by periodically identifying a linear combination of $t$ and $x$. This identification is equivalent to factoring out a discrete subgroup of $S L(2, R)$ [4 [6]. Notice also that the dimensionfull parameter $l$ [2], related to the cosmological constant, can be re-introduced by rescaling $\hat{r}, t$ and $x$. Here it is however taken as unity.

The reason for going through the black string construction is that we want to stress that the limiting process leading to (11) from (8) is somewhat ambiguous. For any finite value of $\rho^{2}$ the spacetime (8) is asymptotically flat, but has string-like curvature singularities surrounded by horizons. The spacetime (11) is however completely different: it is not asymptotically flat and it has no curvature singularities, but there is a horizon at $\hat{r}^{2}=1$. The disappearance of the singularity is understood from the WZW model point of view. This is because the background configuration is obtained from the ungauged $S L(2, R)$ in the limit $\rho^{2}=\infty$; the gauging procedure of $S L(2, R)$ is necessary to produce the singularity [9]. Concerning the thermodynamical properties, we also find some subleties related to taking the limit $\rho^{2}=\infty$. In this limit, the black string is equivalent to the extremal black string, whose statistical temperature is zero. On the other hand, the black hole (11) has a non-vanishing statistical temperature given by the inverse period of the 
imaginary time [2]:

$$
T_{s t}=\frac{1}{2 \pi} \text {. }
$$

So although the black hole anti de Sitter line element (11) comes out in the desired form, by taking the limit $\rho^{2}=\infty$ of the black string, more care is needed for various quantities when it comes to the more detailed analysis of the features of the black hole. In our further analysis we therefore simply take the line element (11), for the $2+1$ dimensional black hole anti de Sitter spacetime, as the starting point.

\section{Tachyon Scattering off the Black Hole}

We now consider the tachyon equation (3) in the background (11). The eigenvalue of the $L_{0}$-operator is:

$$
L_{0} T=\bar{L}_{0} T=-\frac{j(j+1)}{k-2} T,
$$

where we take $j=-1 / 2+i \lambda$ ( $\lambda$ real), which is the principal series representation of $S L(2, R)$. The on-shell condition $L_{0}=1$ (compare with eq. (2)) is obtained as $\lambda^{2}=1 / 92$ for $k=52 / 23$. Then we can solve eq.(3) with eqs.(11) and (13).

The Killing vectors $\partial / \partial_{t}$ and $\partial / \partial_{x}$ suggest that the tachyon field should be solved as:

$$
T(\hat{r}, t, x)=\sum_{N} \int d E T_{E N}(\hat{r}) e^{-i E t} e^{-i N x},
$$

where $N \in Z$ (because of the periodic boundary condition for $x$ ). The tachyon equation then reduces to the following second order ordinary differential equation for $T_{E N}(\hat{r})$ :

$$
\left(\hat{r}^{2}-1\right) \frac{d^{2} T_{E N}}{d \hat{r}^{2}}+\left(3 \hat{r}-\frac{1}{\hat{r}}\right) \frac{d T_{E N}}{d \hat{r}}+\left(\frac{E^{2}}{\hat{r}^{2}-1}-\frac{N^{2}}{\hat{r}^{2}}-4 \lambda^{2}-1\right) T_{E N}=0 .
$$

Further reduction of the problem follows a standard quantum mechanical calculation [8]. Introducing the variable $z \equiv 1-\hat{r}^{2}$ and defining $\Psi_{E N}(z)$ :

$$
T_{E N}(z)=z^{i \epsilon|E| / 2}(1-z)^{i \epsilon^{\prime}|N| / 2} \Psi_{E N}(z)
$$


where $\epsilon, \epsilon^{\prime}= \pm 1$, we find:

$$
z(1-z) \frac{d^{2} \Psi_{E N}}{d z^{2}}+(c-(a+b+1) z) \frac{d \Psi_{E N}}{d z}-a b \Psi_{E N}=0,
$$

where:

$$
\begin{gathered}
a=1 / 2+i \lambda+i\left(\epsilon|E|+\epsilon^{\prime}|N|\right) / 2, \\
b=1 / 2-i \lambda+i\left(\epsilon|E|+\epsilon^{\prime}|N|\right) / 2, \\
c=1+i \epsilon|E| .
\end{gathered}
$$

Equation (17) is the well-known hypergeometric equation. For $|z|<1$ two independent solutions are provided by:

$$
F(a, b, c ; z) \quad \text { and } \quad z^{1-c} F(a-c+1, b-c+1,2-c ; z)
$$

and expressions for $|z| \geq 1$ are obtained using the linear transformation formulas [10]. We will be interested in the two regions $z \rightarrow 0^{-}\left(\hat{r} \rightarrow 1^{+}\right.$; near the horizon) and $z \rightarrow-\infty \quad(\hat{r} \rightarrow \infty$; the asymptotic anti de Sitter region). Near the horizon we find the general solution:

$$
T_{E N}\left(\hat{r} \rightarrow 1^{+}\right) \approx c_{1} e^{i \epsilon|E| \log \sqrt{\hat{r}^{2}-1}}+c_{2} e^{-i \epsilon|E| \log \sqrt{\hat{r}^{2}-1}} .
$$

For $E>0$ and $\epsilon=1$ the first and second terms represent outgoing and ingoing waves, respectively. Similarly we find in the asymptotic region $(\hat{r} \rightarrow$ $\infty)$ :

$$
\begin{aligned}
T_{E N}(\hat{r} \rightarrow \infty) & \approx\left[c_{1} \frac{\Gamma(c) \Gamma(b-a)}{\Gamma(b) \Gamma(c-a)}+c_{2} \frac{\Gamma(2-c) \Gamma(b-a)}{\Gamma(b-c+1) \Gamma(1-a)}\right] e^{-(1+2 i \lambda) \log \hat{r}} \\
& +\left[c_{1} \frac{\Gamma(c) \Gamma(a-b)}{\Gamma(a) \Gamma(c-b)}+c_{2} \frac{\Gamma(2-c) \Gamma(a-b)}{\Gamma(a-c+1) \Gamma(1-b)}\right] e^{-(1-2 i \lambda) \log \hat{\eta}}
\end{aligned}
$$

From these expressions (eqs. (20)-(21)) we now define two different sets of mode solutions. The first set is defined to describe the tachyon scattering off the black hole. Asymptotically it consists of both ingoing and outgoing waves, but at the horizon it is purely ingoing. It is thus obtained by the choices $(E>0) c_{1}=0$ and $\epsilon=1$, and it follows that:

$$
T_{E N}^{(1)}\left(\hat{r} \rightarrow 1^{+}\right) \approx e^{-i E \log \sqrt{\hat{r}^{2}-1}},
$$


as well as:

$$
\begin{aligned}
T_{E N}^{(1)}(\hat{r} \rightarrow \infty) & \approx \frac{\Gamma(2-c) \Gamma(b-a)}{\Gamma(b-c+1) \Gamma(1-a)} e^{-(1+2 i \lambda) \log \hat{r}} \\
& +\frac{\Gamma(2-c) \Gamma(a-b)}{\Gamma(a-c+1) \Gamma(1-b)} e^{-(1-2 i \lambda) \log \hat{r}}
\end{aligned}
$$

and $c_{2}$ has been normalized to 1 . The reflection and transmission coefficients can now be read off immediately:

$$
\begin{aligned}
R & =\left|\frac{\Gamma(a-b) \Gamma(b-c+1) \Gamma(1-a)}{\Gamma(a-c+1) \Gamma(1-b) \Gamma(b-a)}\right|^{2} \\
& =\frac{\cosh \pi(\lambda-(E+N) / 2) \cosh \pi(\lambda-(E-N) / 2)}{\cosh \pi(\lambda+(E-N) / 2) \cosh \pi(\lambda+(E+N) / 2)}, \\
T & =\frac{E}{2 \lambda}\left|\frac{\Gamma(b-c+1) \Gamma(1-a)}{\Gamma(2-c) \Gamma(b-a)}\right|^{2} \\
& =\frac{\sinh \pi E \sinh 2 \pi \lambda}{\cosh \pi(\lambda+(E+N) / 2) \cosh \pi(\lambda+(E-N) / 2)} .
\end{aligned}
$$

and $R+T=1$, as it should. It is interesting to consider the reflection coefficient as a function of $\lambda$, which can be interpreted as the momentum in the $r$-direction. In the two limits we find:

$$
R(\lambda=0)=1, \quad R(\lambda \rightarrow \infty)=e^{-2 \pi E} .
$$

In order to help the understanding of the scattering, typical plots of $R(\lambda)$ for various values of $E$ and $N$ are presented in Figs.1,2. From Fig.1, it can be seen that the reflection coefficient shows a steep fall-off towards the asymptotic value $e^{-2 \pi E}$ for any $E$. The reflected part, which is observed at "large" $\lambda$ (after the fall-off) and represented by

$$
R(\lambda) \approx e^{-2 \pi E}=e^{-E / T s t}
$$

follows the Boltzman distribution with the temperature $T_{s t}=1 / 2 \pi,(12)$.

From Fig.2, we can see the effect of the momentum in the $x$-direction. In order to realize the complete absorption, larger $r$-momentum is necessary 
for larger $x$-momentum, to overcome the propagation of the tachyon in the $x$-direction. Notice that by solving the equation:

$$
\frac{d^{2} R(\lambda)}{d \lambda^{2}}=0
$$

we can, for given $(E, N)$, determine the critical value $\lambda_{\text {crit }}$. where, for $\lambda>$ $\lambda_{\text {crit. }}$, the outgoing wave essentially follows the distribution (27).

Let us now introduce another set of mode solutions that will be relevant to see the Hawking radiation. Asymptotically it will be purely ingoing, but near the horizon it has both outgoing and ingoing parts. It is obtained by the choices $(E>0, \lambda>0) \epsilon=1$ and:

$$
c_{1} \frac{\Gamma(c) \Gamma(a-b)}{\Gamma(a) \Gamma(c-b)}+c_{2} \frac{\Gamma(2-c) \Gamma(a-b)}{\Gamma(a-c+1) \Gamma(1-b)}=0
$$

It follows that:

$$
T_{E N}^{(2)}\left(\hat{r} \rightarrow 1^{+}\right) \approx c_{1} e^{i E \log \sqrt{\hat{r}^{2}-1}}+c_{2} e^{-i E \log \sqrt{\hat{r}^{2}-1}},
$$

as well as:

$$
T_{E N}^{(2)}(\hat{r} \rightarrow \infty) \approx\left[c_{1} \frac{\Gamma(c) \Gamma(b-a)}{\Gamma(b) \Gamma(c-a)}+c_{2} \frac{\Gamma(2-c) \Gamma(b-a)}{\Gamma(b-c+1) \Gamma(1-a)}\right] e^{-(1+2 i \lambda) \log \hat{r}}
$$

The two sets $T_{E N}^{(1)}(\hat{r})$ and $T_{E N}^{(2)}(\hat{r})$ are related by the Bogolubov transformation:

$$
T_{E N}^{(2)}(\hat{r} ; \lambda, E, N)=c_{2} T_{E N}^{(1)}(\hat{r} ; \lambda, E, N)+c_{1} T_{E N}^{*(1)}(\hat{r} ;-\lambda, E, N)
$$

and it is then a standard calculation [11] to evaluate the expectation value of the number operator $N^{(1)}$ in the vacuum state $\mid 0>_{(2)}$ :

$$
{ }_{(2)}<0\left|N^{(1)}\right| 0>_{(2)}=\left(\left|\frac{c_{2}}{c_{1}}\right|^{2}-1\right)^{-1}=\frac{R}{1-R},
$$

where $R$ is given in eq. (24). Finally we define the Hawking temperature by:

$$
{ }_{(2)}<0\left|N^{(1)}\right| 0>_{(2)}=\left(e^{\frac{E}{T_{\text {Hawk. }}}}-1\right)^{-1}
$$


We obtain:

$$
T_{\text {Hawk. }}=E\left[\log \frac{\cosh \pi(\lambda+(E-N) / 2) \cosh \pi(\lambda+(E+N) / 2)}{\cosh \pi(\lambda-(E+N) / 2) \cosh \pi(\lambda-(E-N) / 2)}\right]^{-1},
$$

and for $\lambda>>(E, N)$ we find:

$$
T_{\text {Hawk. }} \approx \frac{1}{2 \pi}
$$

in agreement with the statistical value (12). This is consistent with the above analysis of the reflection coefficient $R$. The region where eq. (36) is valid is where the reflection coefficient $R$ can be approximated by its asymptotic form $\exp (-E / 2 \pi)$. In this region the classical thermodynamical equiliburium is realized. The well-defined thermodynamical picture is obtained in the limit of large momentum in the $r$-direction, where we can understand the problem according to the classical mechanics.

\section{Summary}

The conformally exact form of the $2+1$ dimensional black hole solution is given according to the procedure developed in references [5, 6], starting from the black string solution [7]. The solution obtained in this way, however, is very different from the original black string in the spacetime structure, and it has different thermodynamical properties. Through the analysis of the scattering of a tachyon off the black hole, we find that it is possible to recover the classical picture for large radial momentum of the tachyon. We observe the black body radiation of high momentum particles with the statistical temperature $T_{s t}=1 / 2 \pi$. Our results have been obtained for the $(M=1, J=0)$ black hole anti de Sitter spacetime, but it is straightforward to generalize them to the full $(M, J)$ case. 


\section{References}

[1] E. Witten, Phys. Rev. D44 (1991) 314.

[2] M. Bañados, C. Teitelboim and J. Zanelli, Phys. Rev. Lett. 69 (1992) 1849.

[3] J. Horne and G.T. Horowitz, Nucl. Phys. B368 (1992) 444.

[4] G.T. Horowitz and D.L. Welch, Phys. Rev. Lett. 71 (1993) 328.

[5] R. Dijkgraff, H. Verlinde and E. Verlinde, nucl. Phys. B371 (1992) 269.

[6] N. Kaloper, Phys. Rev. D48 (1993) 2598.

[7] K. Sfetsos, Nucl. Phys. B389 (1993) 424.

[8] S. Flügge, Practical Quantum Mechanics I (Springer-Verlag, New York, 1971) p. 94-99.

[9] P. Ginsparg and F. Quevedo, Nucl. Phys. B385 (1992) 527.

[10] M. Abramowitz and I.A. Stegun, Handbook of Mathematical Functions (Dover Publications Inc., New York, ninth printing, 1970).

[11] N.D. Birrell and P.C.W. Davies, Quantum Fields in Curved Space (Cambridge University Press, Cambridge, 1982). 


\section{Figure Captions}

Fig.1. The reflection coefficient $R(\lambda)$ for $N=3$ and (a) $E=0.1$, (b) $E=0.2$, (c) $E=1$. For "large" $\lambda$ we recover the black-body radiation at temperature $T_{s t}=1 / 2 \pi$.

Fig.2. The reflection coefficient $R(\lambda)$ for $E=1$ and (a) $N=1$, (b) $N=3$, (c) $N=5$. For larger momentum in the $x$-direction we need larger momentum in the $r$-direction to get the same absorption. 
This figure "fig1-1.png" is available in "png" format from: http://arxiv.org/ps/hep-th/9403008v1 
This figure "fig1-2.png" is available in "png" format from: http://arxiv.org/ps/hep-th/9403008v1 